\title{
NONGRAVITATIONAL DECAY OF COSMIC STRINGS
}

\author{
Mark SREDNICKI and Stefan THEISEN ${ }^{1}$ \\ Department of Physics, University of California, Santa Barbara, CA 93106, USA
}

Received 28 July 1986; revised manuscript received 21 November 1986

\begin{abstract}
We investigate the decay of loops of cosmic string via radiation of nongravitational energy. We show that emission of particles other than gravitons and Goldstone bosons is not significant. We use methods which are considerably more rigorous than those used in the past to draw similar conclusions.
\end{abstract}

Cosmic strings are among the more bizarre objects that may be left over from the earliest moments of the Big Bang [1]. Recently there has been much work exploring the possibility that loops of cosmic string were the original seeds for galaxies and clusters of galaxies [1,2], rather than primordial fluctuations in dark matter [3]. Central to this scenario is the idea that a loop of string will decay only by gravitational radiation. It has been clearly established [4] that a loop of string with mass per unit length $\mu$ emits gravitational energy at a rate

$P_{\text {grav }}=\gamma G \mu^{2}$,

where $G$ is Newton's constant and $\gamma$ is a numerical factor depending on the shape (but not the size) of the loop. However, most other emission processes [5] have not been examined with the same level of rigor; we will do so in this letter.

To begin, let $\tilde{\varphi}$ be the superheavy scalar field whose vacuum expectation value is responsible for strings; classically, $\tilde{\varphi}=0$ at the center of the string, but $|\tilde{\varphi}|=V \neq 0$ far from a string. Define a real valued field $\varphi=V-|\tilde{\varphi}|$. Then, far from a string, $\varphi=0$ classically. Suppose a quantum state $|S\rangle$, normalized as $\langle\mathrm{S} \mid \mathrm{S}\rangle=1$, describes an infinite length of string along the $z$-axis; then we have

$\langle\mathbf{S}|\varphi(x, t)| \mathbf{S}\rangle=V f\left(x^{2}+y^{2}\right)$.

1 Address after October 1, 1986: Max-Planck-Institut für Physik and Astrophysik, Werner-Heisenberg-Institut für Physik, Föhringer Ring 6, D-8000 Munich 40, Fed. Rep. Germany.
Here $f\left(\rho^{2}\right)$ is a dimensionless function which is one for $\rho=0$ but falls off rapidly for $\rho \gg a$, where $a \sim 1 / V$ is the thickness of the string. We will approximate $f$ by a delta function:

$$
\langle\mathrm{S}|\varphi(\boldsymbol{x}, t)| \mathrm{S}\rangle \simeq V a^{2} \delta(x) \delta(y) .
$$

For a state describing a closed string located along a general curve, it is necessary to start from a reparameterization invariant expression ${ }^{\ddagger 1}$. We describe the string location with a four-vector $s^{\mu}$ which is a function of two parameters, $\sigma$ and $\tau$. The generalization of eq. (3) is

$$
\begin{aligned}
& \langle\mathrm{S}|\varphi(\boldsymbol{x}, t)| \mathrm{S}\rangle \simeq V a^{2} \int \mathrm{d} \sigma \mathrm{d} \tau(-\operatorname{det} g)^{1 / 2} \\
& \quad \times \delta^{4}\left(x^{\mu}-s^{\mu}(\sigma, \tau)\right),
\end{aligned}
$$

where

$\operatorname{det} g=\left(\frac{\partial s^{\mu}}{\partial \sigma} \frac{\partial s_{\mu}}{\partial \sigma}\right)\left(\frac{\partial s^{\nu}}{\partial \tau} \frac{\partial s_{\nu}}{\partial \tau}\right)-\left(\frac{\partial s^{\mu}}{\partial \sigma} \frac{\partial s_{\mu}}{\partial \tau}\right)^{2}$.

In the center-of-mass frame for the string loop, $s^{0}(\sigma$, $\tau)=\tau$ [6]. Then $\tau=t$, and $s(\sigma, t)$ obeys the equations $[6,7]$

$\ddot{s}=s^{\prime \prime}, \quad\left(\dot{s} \pm s^{\prime}\right)^{2}=1$,

$\boldsymbol{s}(\sigma, t)=\boldsymbol{s}(\sigma+L, t)$,

\$1 We are grateful to A. Vilenkin for bringing this to our attention. An earlier version of this paper had an incorrect form for eq. (7). 
where a prime denotes $\partial / \partial \sigma$ and a dot $\partial / \partial t$. The total mass-energy of the string is $M=\mu L$, where $\mu \sim V^{2}$ is the mass per unit length. Eq. (4) becomes

$$
\begin{aligned}
& \langle\mathrm{S}|\varphi(\boldsymbol{x}, t)| \mathrm{S}\rangle \simeq V a^{2} \int_{0}^{L} \mathrm{~d} \sigma\left|s^{\prime}(\sigma, t)\right|^{2} \\
& \times \delta^{3}(\boldsymbol{x}-s(\sigma, t)) .
\end{aligned}
$$

Powers of $\varphi$ have similar expectation values:

$$
\begin{aligned}
& \left\langle\mathrm{S}\left|\varphi^{n}(x, t)\right| \mathrm{S}\right\rangle \simeq V^{n} a^{2} \int_{0}^{L} \mathrm{~d} \sigma\left|s^{\prime}(\sigma, t)\right|^{2} \\
& \times \delta^{3}(x-s(\sigma, t)) .
\end{aligned}
$$

The string will couple to various other fields. Consider, for example, the field $h$ of the physical Weinberg-Salam Higgs particle. In general the lagrangian will contain interaction terms like $\varphi^{2} h, \varphi h^{2}$, and $\varphi^{2} h^{2}$. Let us use this last interaction to compute, via $L S Z$ reduction, the amplitude for a string state $|S\rangle$ to emit two Higgs particles and become a state $\left|\mathbf{S}^{\prime}\right\rangle$ :

$$
\begin{aligned}
& \left\langle\mathrm{S}^{\prime} ; \mathrm{h}_{1}, \mathrm{~h}_{2} \mid \mathrm{S}\right\rangle \simeq \int \mathrm{d}^{4} x \exp \left(\mathrm{i} k_{1} \cdot x\right) \\
& \quad \times\left\langle\mathrm{S}^{\prime} ; \mathrm{h}_{2}\left|\left(\square+m_{\mathrm{h}}^{2}\right) \mathrm{h}(x)\right| \mathrm{S}\right\rangle \\
& \simeq \int \mathrm{d}^{4} x \exp (\mathrm{i} k \cdot x)\left\langle\mathrm{S}^{\prime} ; \mathrm{h}_{2}\left|\varphi^{2}(x) \mathrm{h}(x)\right| \mathrm{S}\right\rangle \\
& \quad \simeq \int \mathrm{d}^{4} x \exp \left[\mathrm{i}\left(k_{1}+k_{2}\right) \cdot x\right]\left\langle\mathrm{S}^{\prime}\left|\varphi^{2}(x)\right| \mathrm{S}\right\rangle .
\end{aligned}
$$

Here and throughout we do not write coupling constants explicitly. We now assume that the emission had a negligible impact on the string and replace $\left\langle\mathrm{S}^{\prime}\right|$ by $\langle\mathrm{S}|$. Then, using eq. (8), we get

$$
\begin{aligned}
& \left\langle\mathbf{S} ; \mathrm{h}_{1}, \mathrm{~h}_{2} \mid \mathrm{S}\right\rangle \simeq V^{2} a^{2} \int \mathrm{d}^{4} x \exp \left[\mathrm{i}\left(k_{1}+k_{2}\right) \cdot x\right] \\
& \quad \times \int_{0}^{L} \mathrm{~d} \sigma\left|s^{\prime}(\sigma, t)\right|^{2} \delta^{3}(x-s(\sigma, t)) .
\end{aligned}
$$

The prefactor $V^{2} a^{2}$ is of order one; we will ignore it. Now define $E=k_{1}^{0}+k_{2}^{0}$ and $\boldsymbol{k}=\boldsymbol{k}_{1}+\boldsymbol{k}_{2}$, and integrate over $\mathrm{d}^{3} x$ :

$$
\begin{aligned}
& \left\langle\mathrm{S} ; \mathrm{h}_{1}, \mathrm{~h}_{2} \mid \mathrm{S}\right\rangle \simeq \int_{-\infty}^{+\infty} \mathrm{d} t \exp (\mathrm{i} E t) \\
& \quad \times \int_{0}^{L} d \sigma\left|\boldsymbol{s}^{\prime}(\sigma, t)\right|^{2} \exp [-\mathrm{i} \boldsymbol{k} \cdot s(\sigma, t)] .
\end{aligned}
$$

In order to perform the remaining integrals, we need more information about $s(\sigma, t)$. The general solution to eq. (6) is [7]

$$
\begin{aligned}
& \boldsymbol{s}(\sigma, t)=\frac{1}{2} \boldsymbol{a}(\sigma+t)+\frac{1}{2} \boldsymbol{b}(\sigma-t), \\
& \left|\boldsymbol{a}^{\prime}\right|=\left|\boldsymbol{b}^{\prime}\right|=1 .
\end{aligned}
$$

It is not hard to prove that the motion is periodic with period $L / 2$ and that at some time some points on the string move at the speed of light [7,1]. (This is not necessarily true for an infinite length of string.) The periodicity means that we can write

$$
\begin{aligned}
& \int_{0}^{L} \mathrm{~d} \sigma\left|\boldsymbol{s}^{\prime}(\sigma, t)\right|^{2} \exp [-\mathrm{i} \boldsymbol{k} \cdot s(\sigma, t)] \\
& \quad=\sum_{n} a_{n} \exp (-4 \pi \mathrm{i} n t / L),
\end{aligned}
$$

where

$$
\begin{aligned}
a_{n} & =\frac{2}{L} \int_{0}^{L / 2} \mathrm{~d} t \exp (4 \pi \mathrm{int} t / L) \\
& \times \int_{0}^{L} \mathrm{~d} \sigma\left|\boldsymbol{s}^{\prime}(\sigma, t)\right|^{2} \exp [-\mathrm{i} \boldsymbol{k} \cdot \boldsymbol{s}(\sigma, t)]
\end{aligned}
$$

is a function of $k$. This gives

$$
\left\langle\mathrm{S} ; \mathrm{h}_{1}, \mathrm{~h}_{2} \mid \mathrm{S}\right\rangle \simeq 2 \pi \sum_{n} a_{n} \delta(E-4 \pi n / L) \text {. }
$$

The total energy radiated is

$$
P T=\int \widetilde{\mathrm{d} k_{1}} \widetilde{\mathrm{d} k_{2}} E\left|\left\langle\mathrm{~S} ; \mathrm{h}_{1}, \mathrm{~h}_{2} \mid \mathrm{S}\right\rangle\right|^{2},
$$

where $P$ denotes power, $T=2 \pi \delta(0)$, and $\mathrm{d} k=$ $\mathrm{d}^{3} k /(2 \pi)^{3} 2 k^{0}$. Thus 
$P=\sum_{n} P_{n}$,

$P_{n}=2 \pi \int \mathrm{d} k_{1} \mathrm{~d} k_{2} E\left|a_{n}\right|^{2} \delta(E-4 \pi n / L)$.

At this point it is convenient to use units such that $L=1$; since we are contemplating cosmic strings, this means that our unit of length is very large and our unit of mass is very small (by particle physics standards). We must still compute $a_{n}$; setting $E=4 \pi n$,

$$
\begin{aligned}
a_{n} & =2 \int_{0}^{1 / 2} \mathrm{~d} t \int_{0}^{1} \mathrm{~d} \sigma\left|\boldsymbol{s}^{\prime}(\sigma, t)\right|^{2} \\
& \times \exp \{\mathrm{i}[E t-\boldsymbol{k} \cdot \boldsymbol{s}(\sigma, t)]\} .
\end{aligned}
$$

We can evaluate $a_{n}$ by the method of stationary phase. Define

$\psi=E t-\boldsymbol{k} \cdot \boldsymbol{s}(\sigma, t)$.

Clearly $\psi^{\prime}=\dot{\psi}=0$ only if $s^{\prime}=0$ and $|\dot{s}|=1$, but we already know this is true for some $\sigma$ and $t$. Choose coordinates so that at this point $\sigma=t=0, s=0$, and $\dot{s}=\boldsymbol{z}$. Then we also need $\boldsymbol{k}=E \hat{z}$ to have $\psi^{\prime}=\dot{\psi}=0$. This means $\boldsymbol{k}_{1}=k_{1}^{0} \hat{z}$ and $\boldsymbol{k}_{2}=k_{1}^{0} \hat{z}$, which is only one point in phase space, and possible only if $m_{\mathrm{h}}=0$. In a moment we will see that we can relax these conditions, but let us keep them for now. The value of the integral will then be given approximately by integrating $\left|s^{\prime}\right|^{2}$ over the area of the $\sigma, t$ plane which corresponds to $|\psi| \lesssim 1$. Using eq. (12) it is easy to show that for small $\sigma$ and $t, s(\sigma, t)$ has a power series expansion of the form

$s_{z}=t+c_{1}\left(t^{3}+3 t \sigma^{2}\right)+c_{2}\left(\sigma^{3}+3 \sigma t^{2}\right)+\ldots$,

$s_{y}=c_{3}\left(t^{2}+\sigma^{2}\right)+2 c_{\mathrm{A}} \sigma t+\ldots$,

$s_{x}=c_{5}\left(t^{2}+\sigma^{2}\right)+2 c_{6} \sigma t+\ldots$.

In general, the coefficients $c_{i}$ are all of order one, but they are not independent. Using the expansion for $s_{z}$ in eq. (19) we see that $|\psi|$ will remain less than order one provided the absolute values of the following expressions are all less than order one:

$E t^{3}, E t^{2} \sigma, E t \sigma^{2}, E \sigma^{3}$.

This determines the maximum values of $\sigma$ and $t$ before rapid oscillation begins: $t_{\max } \sim \sigma_{\max } \sim E^{-1 / 3}$.

Also, we see from eq. (20) that $\left|s^{\prime}(\sigma, t)\right|^{2}$ has terms of order $\sigma^{2}, \sigma t$, and $t^{2}$. Therefore we estimate

$$
\left|a_{n}\right| \sim \int_{-\sigma_{\max }}^{\sigma_{\max }} \mathrm{d} \sigma \int_{-t_{\max }}^{t_{\max }} \mathrm{d} t\left(\sigma^{2}, \sigma t, t^{2}\right) \sim E^{-3 / 4} .
$$

We now note that the stationary phase approximation will remain valid if we add a term linear in $t$ provided that for $t=t_{\max }$ this term is less than order one. Hence we can allow $\boldsymbol{k}_{1}$ and $\boldsymbol{k}_{2}$ to lie within a small angle $\epsilon$ of $z$. Then to leading order in $\epsilon$ and $m_{\mathrm{h}}$,

$E-k_{z} \sim m_{\mathrm{h}}^{2} E^{-1}+E \epsilon^{2}, \quad k_{x}, k_{y} \sim E \epsilon$.

We have the new conditions that each of the following expressions have absolute value less than order one:

$m_{\mathrm{h}}^{2} E^{-1} t_{\max }, E \epsilon^{2} t_{\max }, E \epsilon t_{\max }^{2}$,

$E \epsilon t_{\max } \sigma_{\max }, E \epsilon \sigma_{\max }^{2}$.

The last four of these restrictions yield, for fixed $E$,

$\epsilon_{\max } \sim E^{-1 / 3}$,

while the first gives

$E_{\min } \sim m_{\mathrm{h}}^{3 / 2}$.

Thus eq. (23) remains valid for $\epsilon<\epsilon_{\max }$, and $E>E_{\min }$.

We now use all this in the formula for $P_{n}$, eq. (17):

$$
\begin{aligned}
P_{n} & =2 \pi \int \widetilde{\mathrm{d} k_{1}} \widetilde{\mathrm{d} k_{2}} E\left|a_{n}\right|^{2} \delta(E-4 \pi n) \\
& \simeq n \int k_{1}^{0} \mathrm{~d} k_{1}^{0} k_{2}^{0} \mathrm{~d} k_{2}^{0} \mathrm{~d} \Omega_{1} \mathrm{~d} \Omega_{2}\left|a_{n}\right|^{2} \\
& \times \delta\left(k_{1}^{0}+k_{2}^{0}-4 \pi n\right) .
\end{aligned}
$$

In the second line we have ignored all numerical factors. Since both $\boldsymbol{k}_{1}$ and $\boldsymbol{k}_{2}$ are within an angle $\epsilon_{\max }$ of $\hat{z}$, we have $\mathrm{d} \Omega_{1} \sim \mathrm{d} \Omega_{2} \sim \epsilon_{\max }^{2}$, and so

$P_{n} \sim n^{4} \epsilon_{\max }^{4}\left|a_{n}\right|^{2} \sim 1$.

Obviously the sum over $n$ will diverge. Of course, our formalism of an infinitely thin string in inadequate to deal with emission of particles with $E \gtrsim V$. Therefore we should cut off the sum at $n \sim V \sim \mu^{1 / 2}$. Provided that $V>E_{\min } \sim m_{\mathrm{h}}^{3 / 2}$, we get

$P \sim(V L)^{-1} \mu$, 
where we have restored appropriate factors of $L$.

This power is negligible compared to the gravitational power of eq. (1). For $V \sim 10^{16} \mathrm{GeV}$ and $L \sim 1$ kpc, $P \sim 10^{-51} \mu$ whereas $P_{\text {grav }} \sim 10^{-5} \mu$ for typical values of $\gamma$. Furthermore, we cannot increase the result by considering emission of other types of particles. If the coupling of $\varphi$ to the associated fields does not involve derivatives, we simply reproduce eq. (30). If there are derivatives, extra factors like $k_{1} \cdot k_{2} / V^{2}$ appear in the matrix element. These factors invariably suppress the power output even more.

One should be aware, though, that our methods are essentially perturbative. For example, they do not uncover the fact that strings resulting from spontaneous breakdown of a global symmetry copiously emit the corresponding Goldstone boson [8]. This effect is due to coherent radiation and is found by making a classical analysis. Thus it is still possible that there may be nonperturbative effects leading to rapid decay of string loops into particles other than gravitons or (in the case of global symmetry breaking) Goldstone bosons. We hope to return to this question in the future. For now, though, our conclusion confirms the conventional wisdom [5] that strings resulting from the breakdown of a local symmetry disappear only by gravitational radiation.
We acknowledge helpful discussions with D.P. Bennett and K.A. Olive. We thank A. Vilenkin for suggesting eq. (4) as the proper starting point. This work was supported in part by the National Science Foundation under Grant No. PHY83-13324.

\section{References}

[1] A. Vilenkin, Phys. Rep. 121 (1985) 263, and references therein.

[2] A. Albrecht and N. Turok, Phys. Rev. Lett. 54 (1985) 1868; N. Turok, Phys. Rev. Lett. 55 (1985) 1801;

D.P. Bennett, Phys. Rev. D 33 (1986) 872;

R. Brandenburger and N. Turok, Phys. Rev. D 33 (1986) 2175.

[3] J. Primack, SLAC-PUB-3387, lectures presented Intern. School of Physics "Enrico Fermi" (Varenna, Italy, June 26-July 6, 1984).

[4] T. Vachaspati and A. Vilenkin, Phys. Rev. D 31 (1985) 3052; C.J. Burden, Phys. Lett. B 164 (1985) 277.

[5] T. Vachaspati, A.E. Everett and A. Vilenkin, Phys. Rev. D 30 (1984) 2046.

[6] P. Goddard, J. Goldstone, C. Rebbi and C.B. Thorn, Nucl. Phys. B 56 (1973) 109.

[7] T.W.B. Kibble and N. Turok, Phys. Lett. B 116 (1982) 141.

[8] R.L. Davis, Phys. Rev. D 32 (1985) 3172. 